Article

\title{
Isolation and Analysis of the Cppsy Gene and Promoter from Chlorella protothecoides CS-41
}

\section{Meiya Li ${ }^{1,2, \dagger}$, Yan Cui ${ }^{1, \dagger}$, Zhibing Gan ${ }^{1}$, Chunlei Shi ${ }^{1, *}$ and Xianming Shi ${ }^{1}$}

1 MOST-USDA Joint Research Center for Food Safety, School of Agriculture and Biology, and State Key Lab of Microbial Metabolism, Shanghai Jiao Tong University, Shanghai 200240, China; E-Mails: 1meiya@126.com (M.L.); cyan9028@163.com (Y.C.); forrestgzb@yahoo.cn (Z.G.); xmshi@sjtu.edu.cn (X.S.)

2 Analytical Testing Center, Zhejiang Chinese Medical University, Hangzhou 310053, China

$\dagger$ These authors contributed equally to this work.

* Author to whom correspondence should be addressed; E-Mail: clshi@sjtu.edu.cn; Tel.: +86-21-3420-5755; Fax: +86-21-3420-6616.

Academic Editor: Graziano Riccioni

Received: 25 June 2015 / Accepted: 9 September 2015 / Published: 28 October 2015

\begin{abstract}
Phytoene synthase (PSY) catalyzes the condensation of two molecules of geranylgeranyl pyrophosphate to form phytoene, the first colorless carotene in the carotenoid biosynthesis pathway. So it is regarded as the crucial enzyme for carotenoid production, and has unsurprisingly been involved in genetic engineering studies of carotenoid production. In this study, the psy gene from Chlorella protothecoides CS-41, designated Cppsy, was cloned using rapid amplification of cDNA ends. The full-length DNA was $2488 \mathrm{bp}$, and the corresponding cDNA was $1143 \mathrm{bp}$, which encoded 380 amino acids. Computational analysis suggested that this protein belongs to the Isoprenoid_Biosyn_C1 superfamily. It contained the consensus sequence, including three predicted substrate- $\mathrm{Mg}^{2+}$ binding sites. The Cppsy gene promoter was also cloned and characterized. Analysis revealed several candidate motifs for the promoter, which exhibited light- and methyl jasmonate (MeJA)-responsive characteristics, as well as some typical domains universally discovered in promoter sequences, such as the TATA-box and CAAT-box. Light- and MeJA treatment showed that the Cppsy expression level was significantly enhanced by light and MeJA. These results provide a basis for genetically modifying the carotenoid biosynthesis pathway in $C$. protothecoides.
\end{abstract}


Keywords: Chlorella protothecoides CS-41; phytoene synthase; Cppsy; promoter

\section{Introduction}

Lutein is one of more than 750 known naturally occurring carotenoids, and is synthesized by higher plants, bacteria, fungi, and algae. Based on its molecular structure (containing oxygen), it belongs to the xanthophyll family, one of the two major carotenoid families. In the plant kingdom, lutein provides photoprotection by scavenging singlet oxygen and peroxyl radicals [1], and its bright yellow color helps plants achieve effective cross pollination. Humans cannot synthesize lutein themselves, yet it is essential for the human body. Lutein is the predominant carotenoid in the infant brain [2], and is supplemented in newborn babies in the first hours of life. Lutein can increase biological antioxidant potential and reduce the plasma concentration of total hydroperoxides. It also reduces free radical-induced damage [3]. Lutein is the main carotenoid in the human retina; hence, it has been used as a therapeutic agent for the prevention of age-related macular degeneration [4,5]. Epidemiologic data suggest that lutein plays an active role in delaying chronic diseases [6], stimulating the immune response [7], and hampering the development of cataracts and atherosclerosis [8,9]. A recent study showed that a lutein-based dye used during chromovitrectomy in humans could improve the identification and removal of the vitreous, internal limiting membrane and the epiretinal membrane [10].

As lutein has many functions, it has become increasingly important to find and create more sources of lutein production. In recent years, algae have received a great deal of attention in the production of carotenoids and proteins. Previous studies in our laboratory showed that heterotrophically cultivated Chlorella protothecoides CS-41 can produce considerable amounts of lutein [11]. Furthermore, optimization of the cultivation conditions, medium composition, and extraction techniques can improve lutein yields $[12,13]$. However, to date, there are no reports of the enhancement of lutein production by this alga using genetic modification, although genetic engineering technologies have become increasingly popular in the field of carotenoid production. The first step is to determine the genes involved in lutein biosynthesis - information that is essential for genetic modification.

It has been found that phytoene synthase (PSY) is the rate-limiting enzyme in the carotenoid biosynthesis pathway in photosynthetic organisms [14-16]. In many cases, the rate of lutein formation through the carotenoid biosynthetic pathway appears to be controlled by PSY, which catalyzes the head-to-head condensation of two geranylgeranyl diphosphate molecules to yield phytoene - the first committed reaction in carotenogenesis. Since PSY plays a key role in the first step of carotenogenesis, it has unsurprisingly been chosen for genetic engineering studies of carotenoid production.

PSY has been extensively studied in bacteria and higher plants, but its study in algae is still in its infancy. For unicellular green algae, the psy gene has previously been investigated in Chlamydomonas reinhardtii [17], Duanliella bardawil [18], and Haematococcus pluvialis [19]. In C. reinhardtii, deletion of the psy gene resulted in a white phenotype [20]. For Haematococcus, psy was shown to be up-regulated under stress conditions of high light and low nutrient availability [21]. Overexpression of 
exogenous psy from D. salina [22] or C. zofingiensis [23] in C. reinhardtii has been shown to increase the lutein content to over 1.25 and 2.2-fold, respectively.

As an efficient lutein-production alga, $C$. protothecoides CS-41 has high potential for application in the commercial production of lutein; however, its lutein biosynthesis pathway has not been well studied. Our research group previously cloned other key enzyme genes in the lutein biosynthesis pathway of this alga, such as the phytoene desaturase ( $p d s$ ) (GenBank accession No. FJ968162) [24], zeta-carotene desaturase ( $z d s$ ) (GenBank accession No. GU269622) [25], and lycopene- $\varepsilon$-cyclase (lyce) (GenBank accession No. FJ752528) genes. The psy gene is essential for determining the complete lutein biosynthesis pathway in this alga. Therefore, in this study, the psy gene from the unicellular microalga $C$. protothecoides CS-41 and its promoter were isolated and analyzed. This study provides an important theoretical basis for the genetic modification of lutein biosynthesis in C. protothecoides CS-41, including gene sequences, expression promoter candidates, and possible regulatory environmental factors for gene expression.

\section{Results and Discussion}

\subsection{Cloning and Characterization of the psy Gene from C. protothecoides}

Touchdown PCR with primers YF and YR (Table 1) generated a predicted 373 bp fragment (Supplementary Figure S1, lane 1). BLAST analysis showed that the nucleotide sequence of this fragment shared about $74 \%$ and $73 \%$ identities with that of C. reinhardtii and D. salina, respectively, demonstrating that this fragment sequence is derived from a putative phytoene synthase.

Table 1. PCR primers and target fragments for Cppsy. F: forward; R: reverse; O: outer primer; I: inner primer.

\begin{tabular}{cc}
\hline Aim & Primer Sequence 5' $\mathbf{- 3}$ \\
\hline Partial psy fragment & \\
\hline YF & GCCATCTACGTGTGGTGCC \\
YR & CACGCAAGATGTTGGTCAGC \\
\hline $\mathbf{5}^{\prime}$-RACE-PCR & \\
\hline YFO1 & GACTTGTCCACGCCCATCAC \\
YRI1 & GGGGAAGCGGGAGATGGTGT \\
\hline 3'-RACE-PCR & \\
\hline YFO2 & GATGCTGCCCTCACAGACAC \\
YRI2 & TGGATTTGGTCAAGTCACGC \\
\hline cDNA and DNA & \\
\hline YF1 & \\
YR1 & ATGAGCACGTTTCTGAGCACAGTG \\
\hline Probe & TCACATGCGCGCCCTCAG \\
\hline psy-F & \\
psy-R & GAAGTGACCAGCGAGTATGCC \\
psyRTF & CTAAAGGGTTGGATGTGC \\
psyRTR & GAAGTGACCAGCGAGTATGCC \\
\hline
\end{tabular}


Table 1. Cont.

\begin{tabular}{cr}
\hline Promoter & \\
\hline PSYSP1 & CTGTGCATGCGAAGTCGGAGTGAGA \\
PSYSP2 & CGTCTTGGCATACTCGCTGGTCACTT \\
PSYSP3 & ACTCATGCTGGGGGCTAGGAAAG \\
PSYSP1' & ATGGCGGGTGGCAGAGTCAATGTA C \\
PSYSP2 & CCAGACACAATCACCTCGCAGCCCTT \\
PSYSP3' & CGTTCACTCACCGCTCTCCATCACAA \\
\hline
\end{tabular}

With the sequence information, specific primers were designed for 5'- and 3'-rapid amplification of cDNA ends (RACE) of the related gene. 5'-RACE generated a 598 bp fragment (Supplementary Figure S1, lane 2), and 3'-RACE produced an 816 bp fragment (Supplementary Figure S1, lane 3). They were displayed by sequencing as the $5^{\prime}$ and $3^{\prime}$ regions of the phytoene synthase gene of $C$. protothecoides (Cppsy). RT-PCR (Reverse Transcription) with a pair of primers YF1 and YR1 generated an 1143 bp fragment (Supplementary Figure S1, lane 4), which was identified as the full-length Cppsy cDNA (GenBank accession No. FJ968161).

The open reading frame of Cppsy cDNA encoded a protein of 380 amino acid residues with a calculated molecular mass of $43.035 \mathrm{kDa}$ and an isoelectric point of 6.40 (http://cn.expasy.org/ tools/protparam.html) and shared $81.7 \%$ identical sequence with Chlorella NC_64A.

To characterize the corresponding gene of Cppsy cDNA, genomic PCR was performed. A 2488 bp fragment (Supplementary Figure S1, lane5) (GenBank accession No. GU351883) was generated and sequenced. Analysis of the obtained nucleotide sequence revealed that the product was the corresponding Cppsy gene.

The Southern blot analysis results indicated that there is only one Cppsy gene copy in C. protothecoides CS-41 (Supplementary Figure S2), which is different to those in higher plants. Psy gene replication is common in dicot plants, such as tomato (SIPSY1 and SIPSY2), and in monocot plants, such as maize (ZmPSY1-3), rice (OsPSY1-3), and sorghum (SbPSY1-3) [16,26-28].

Analysis of the Cppsy gene structure (Figure 1) revealed that it is more complicated than those of dicot and monocot plants. It consists of ten exons and nine introns. Chlorella has a higher intron density than other algae and higher plants; in most of the higher plants, psy genes always have four or five introns, but this alga has nine introns. Compared with the structure of the psy gene from C. reinhardtii (Crpsy), it seems that there are two introns inserted into each of the first and second exons, and one intron inserted into the fourth exon, which makes the gene structure more complicated (Figure 1C). 


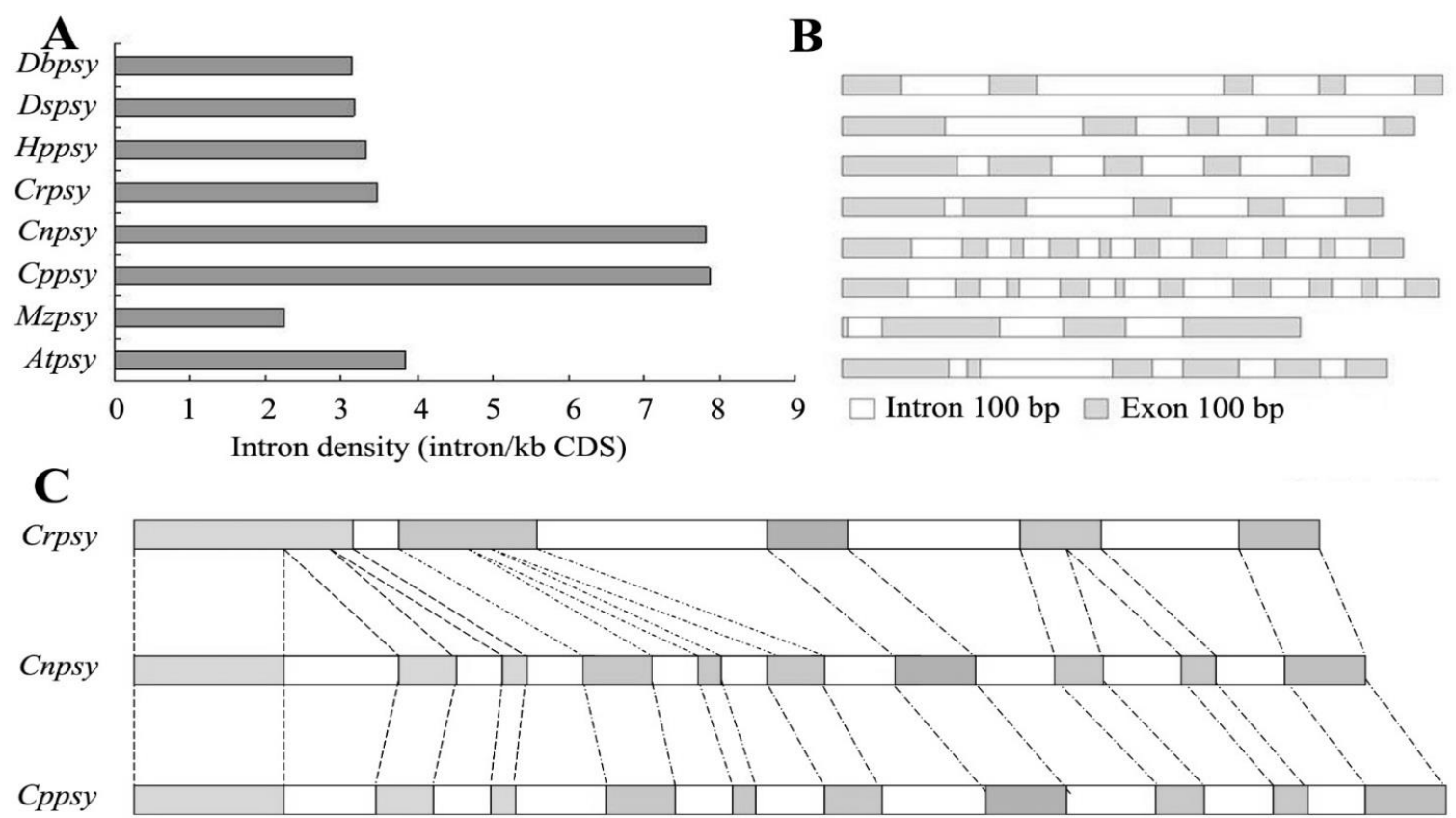

Figure 1. Exons and introns of the Cppsy gene in C. protothecoides CS-41.The ten exons are: (1) $1 \mathrm{bp}$ to $280 \mathrm{bp}$; (2) $477 \mathrm{bp}$ to $576 \mathrm{bp}$; (3) $691 \mathrm{bp}$ to $743 \mathrm{bp}$; (4) $913 \mathrm{bp}$ to $1030 \mathrm{bp}$; (5) $1139 \mathrm{bp}$ to $1180 \mathrm{bp}$; (6) $1327 \mathrm{bp}$ to $1427 \mathrm{bp}$; (7) $1635 \mathrm{bp}$ to $1793 \mathrm{bp}$; (8) $1957 \mathrm{bp}$ to $2045 \mathrm{bp}$; (9) 2171 bp to $2233 \mathrm{bp}$; (10) 2351 bp to 2488 bp. (A) Intron density; (B) DNA structure; (C) The relationship between introns and exons of Cppsy, Crpsy, and Cnpsy genes. Dbpsy, Dspsy, Hppsy, Crpsy, Cnpsy, Cppsy, Mzpsy, and Atpsy are the psy genes of Duanliella bardawil, Duanliella salina, Haematococcus pluvialis, Chlamydomonas reinhardtii, Chlorella NC_64A, Chlorella protothecoides CS-41, Zea mays, and Arabidopsis thaliana, respectively.

\subsection{Sequence Alignment and Phylogenetic Reconstruction}

After the DNA and cDNA sequences of the Cppsy gene were determined, it was possible to investigate its evolutionary position among the various psy genes. Using MEGA 4.0 from Clustal W1.6 alignments, the phylogenetic tree of PSYs from different organisms was constructed based on their deduced amino acid sequences. It showed that psy was derived from an ancestor gene and later evolved into four subgroups, including higher plants, cyanobacteria, algae, and bacteria (Figure 2). According to the neighbor-joining (NJ) tree, Cppsy belongs to the algae group, and is more ancient than plant species (Figure 2).

The deduced amino acid sequence of Cppsy was submitted to NCBI for PSI-BLAST searches and the results showed that Cppsy has high homology with psy genes from other algal species, with $83 \%$ identity and $88 \%$ positives with psy from Chlorella NC_64A. Cppsy was also highly similar to psy from $C$. reinhardtii (67\% identities, $79 \%$ positives), $H$. pluvialis (63\% identities, $77 \%$ positives), D. bardawil (68\% identities, $80 \%$ positives), and D. salina (68\% identities, $79 \%$ positives), suggesting that Cppsy belongs to the algae psy family. In the algae family, CpPSY belongs to class I of PSY according to Tran's data [29]. BlastP analysis suggested that this protein has the essential characteristics of PSY. It belongs to the Isoprenoid_Biosyn_C1 superfamily, and contains the consensus sequence, including three predicted substrate- $\mathrm{Mg}^{2+}$ binding sites (aspartate-rich regions) (DXXXD), 
130-DELVD-134, 203-DELYD-207, and 256-DEGED-260 (Figure 3A). In other algae and higher plants, there are two (DELVD and DVGED) (Figure 3A); hence, CpPSY has one more DXXXD motif than other PSYs. The abundant 203-DELYD-207 site possibly plays an important role in the function of CpPSY, which should be studied further.

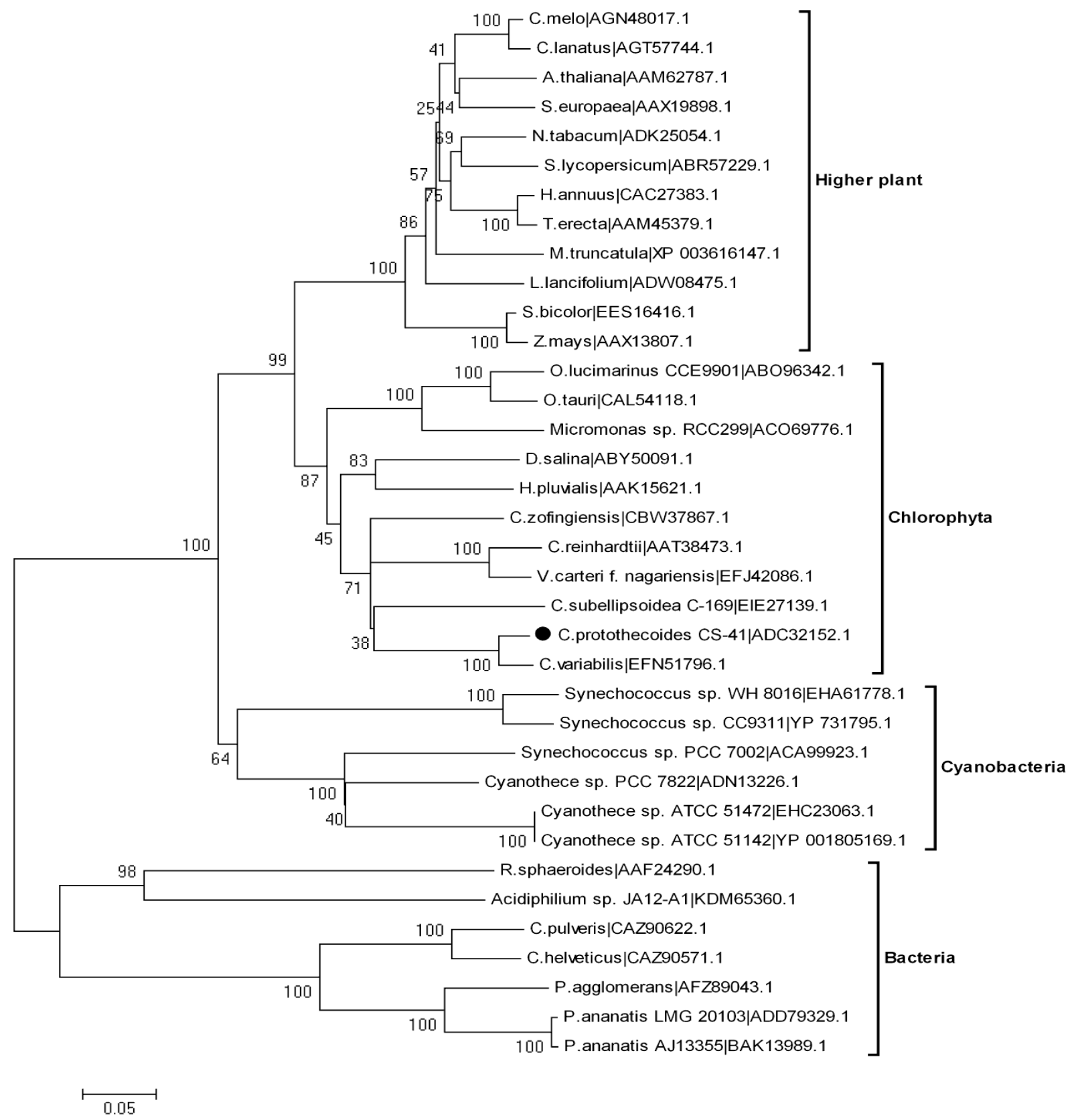

Figure 2. Phylogenetic tree of PSY sequences from various species. The phylogeny was derived using neighbor-joining analysis. The accession numbers of the amino acid sequences follow the taxon names. Horizontal branch lengths represent relative evolutionary distances, with the scale bar corresponding to 0.05 amino acid substitutions per site. 


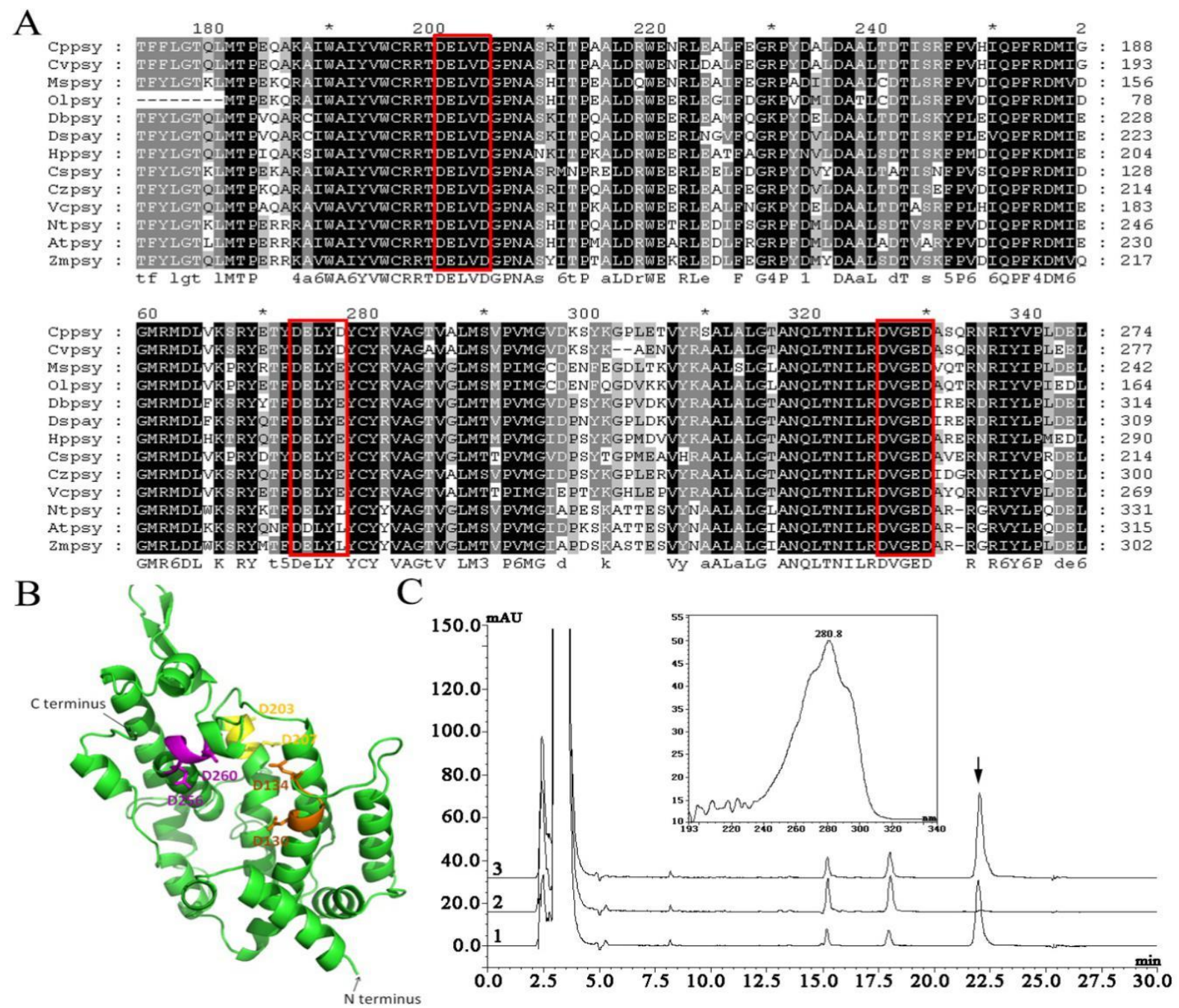

Figure 3. (A) Alignment of the selective PSY-deduced amino acid sequences from different algae produced with the GeneDoc program using Clustal W. The alignment indicates aspartate-rich regions/substrate- $\mathrm{Mg}^{2+}$ binding sites (DXXXD). The three DXXXD motifs are shown by the red boxes. Cppsy, Crpsy, Mspsy, Olpsy, Dbpsy, Dspsy, Hppsy, Cspsy, Czpsy, Vcpsy, Ntpsy, Atpsy, and Zmpsy are the PSY of Chlorella protothecoides CS-41, Chlorella variabilis, Micromonas sp. RCC299, Ostreococcus lucimarinus, Duanliella bardawil, Duanliella salina, Haematococcus pluvialis, Coccomyxa subellipsodiea C-169, Chromochloris zofingiensis, Volvox carteri f. nagariensis, Nicotiana tabacum, Arabidopsis thaliana, and Zea mays, respectively; (B) Three-dimensional model structure of CpPSY. Comparative modeling was performed using homology-based three-dimensional structural modeling. The three aspartate-rich motifs (DXXXD) are colored in orange (DELVD), yellow (DELYD), and magenta (DEGED); others are shown in green. The $N$-terminus and $C$-terminus are also shown; (C) High-performance liquid chromatography trace and UV spectrum of carotenoid pigments in the E. coli heterologous complementation system. Pigments extracted from E. coli cells transformed with pACCRT-E and pUC-psy together (1), pUC-psy only (2), and pACCRT-EB only (3). Absorbance was recorded at $285 \mathrm{~nm}$. The peak indicated by the arrow is phytoene. 
The secondary structure prediction carried out at NPS@ (https://npsa-prabi.ibcp.fr/) showed that CpPSY consists of $58.68 \%$ alpha helix, $26.58 \%$ random coil, $10.79 \%$ extended strand, and $3.95 \%$ beta turn. The tertiary structure of CpPSY was constructed using homology-based modeling by Swiss-Model (Figure 3B). A total of 50 models were found. Squalene synthase (HpnC) was used as a template for molecular modeling, since the identity is the highest (30.42\%). The modeled structure also showed that CpPSY consists mostly of alpha helices. The three conserved DXXXD motifs (orange DELVD, yellow DELYD, and magenta DEGED) were marked in the three-dimensional model structure (Figure 3B). It seems that the three DXXXD motifs form a circle-like structure, which could be important for enzyme activity.

All of the analysis results strongly suggest that PSY from $C$. protothecoides CS-41 is an algal phytoene synthase protein involved in the carotenoid biosynthesis pathway. Bacterial complementation assay further confirmed that this gene is functional. The expressed protein in pUC-psy could catalyze the GGPP produced by pACCRT-E (Figure 3C,1) to phytoene, similar to the function of the $\operatorname{crtB}$ gene in pACCRT-EB (Figure 3C,3).

\subsection{Promoter Isolation and Analysis}

The promoter region of the Cppsy gene was cloned from $C$. protothecoides CS-41 genomic DNA. The cloned Cppsy promoter region was determined to be $1980 \mathrm{bp}$ in length, and the sequence is shown in Figure 4. Furthermore, the cloned Cppsy promoter region was analyzed using the PLACE and PlantCARE databases. Several core fragments were identified, which are homologous to the cis-acting elements of higher plants and of great importance for the promoter functions (Figure 4). Three types of elements, which have been found to be regulated by hormones in the upstream region of some plant genes, are present in the Cppsy promoter: the ABRE type (CCTGCGTGGC, CACGTG, and GCCTCGTGGC) involved in abscisic acid responsiveness; the TGACG-motif (TGACG), the cis-acting regulatory element involved in methyl jasmonate (MeJA) responsiveness; and the Sp1 (CCCCCGCCA and ACCCGCCATG), MNF (GTGCCCCATGCAGGTT) and Box I (TTTCAAA) types involved in light responsiveness.

The transcriptional start site (TSS) of the Cppsy promoter was determined by 5'-RACE using total RNA extracted from C. protothecoides. The TSS is an adenine (A) at 34 bp upstream of the translation initiation codon. The distance between the putative TATA-box and TSS is approximately -24 to $-28 \mathrm{bp}$, which is consistent with the distance of $32 \mathrm{bp} \pm 7 \mathrm{bp}$ from previous data [30].

A previous study showed that the psy expression level is affected by light [31] and other biotic and abiotic stresses [16] in higher plants. Gene expression response to environmental stress is related to the regulation mechanism. To understand more about the regulation mechanism, we need to know more information about the gene, including the gene structure and regulatory domains. Here, many elements were found in the Cppsy promoter that belong to light-responsive elements, such as Sp1, MNF1, G-box, and chs-CMA2a. There were also some cis-acting elements involved in abscisic acid (ABRE) and MeJA (TGACG-motif) responsiveness. Light is one of the most important environmental factors for algae. To determine whether these motif sequences from the Cppsy promoter are involved in light, abscisic acid, and MeJA signaling, loss-of-function analysis needs to be carried out in future studies. 


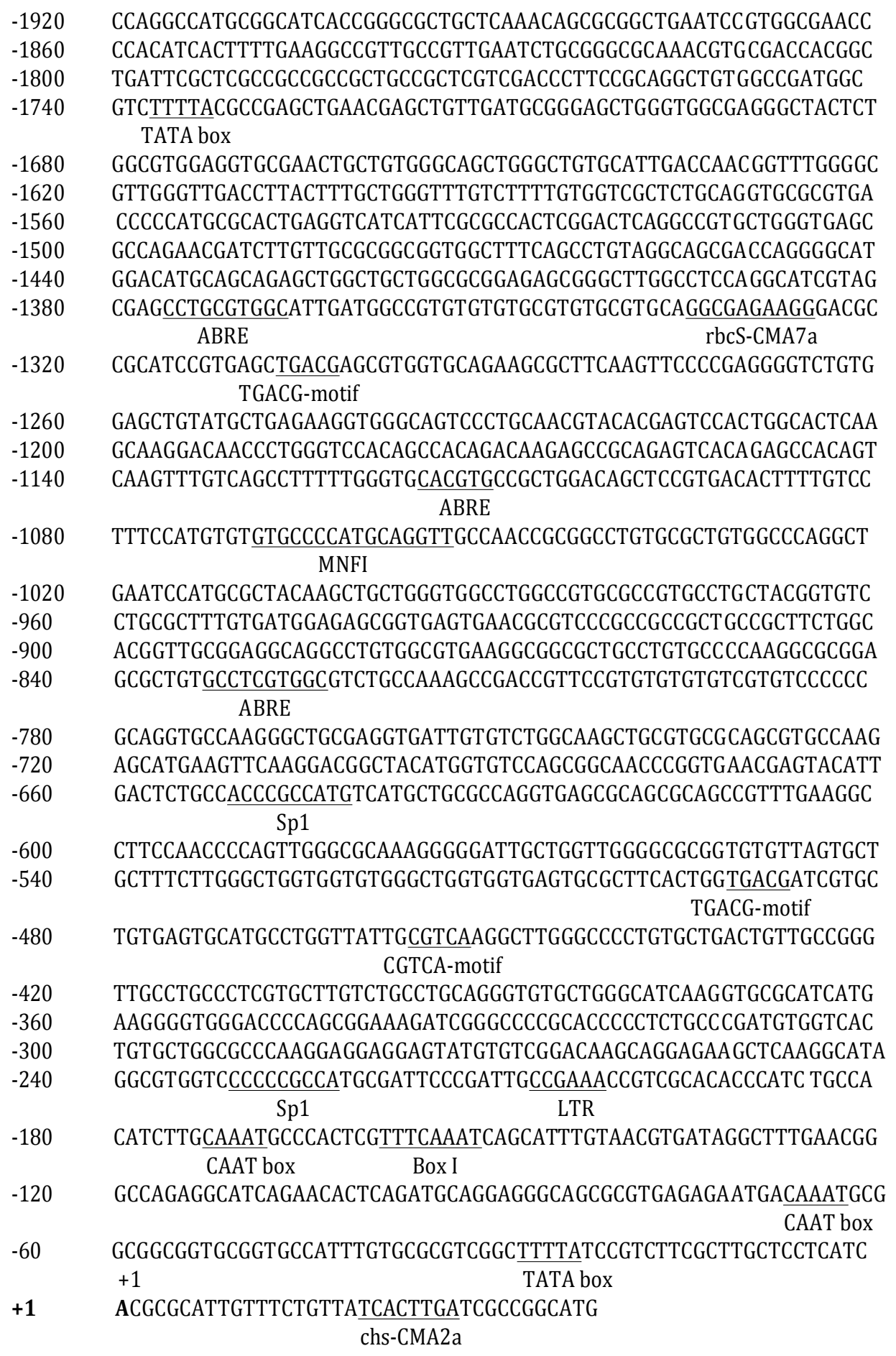

Figure 4. Promoter sequence of Cppsy from C. protothecoides CS-41. Numbers indicate the positions relative to transcriptional start site (TSS). The TSS is indicated as +1 and in bold; important cis-elements are underlined.

\subsection{Gene Expression Response to Light and MeJA}

To investigate the effects of light and MeJA on the promoter inducibility at different time points, we analyzed Cppsy mRNA expression levels after light and MeJA treatment. The results show that on treatment with light, Cppsy gene expression increased by up to 36 times, compared with the dark, which indicates that Cppsy gene expression is up-regulated in response to light (Figure 5A). When 
treated with MeJA, Cppsy gene expression peaked at $10 \mathrm{~h}$ after treatment (Figure 5B). Therefore, the gene expression of Cppsy is significantly induced by treatment with light and MeJA $(p<0.01)$. These results confirm that the Cppsy promoter is induced by light and MeJA, and can be used as a candidate promoter element for the genetic modification of carotenoid biosynthesis in Chlorella, other algae, or higher plants.
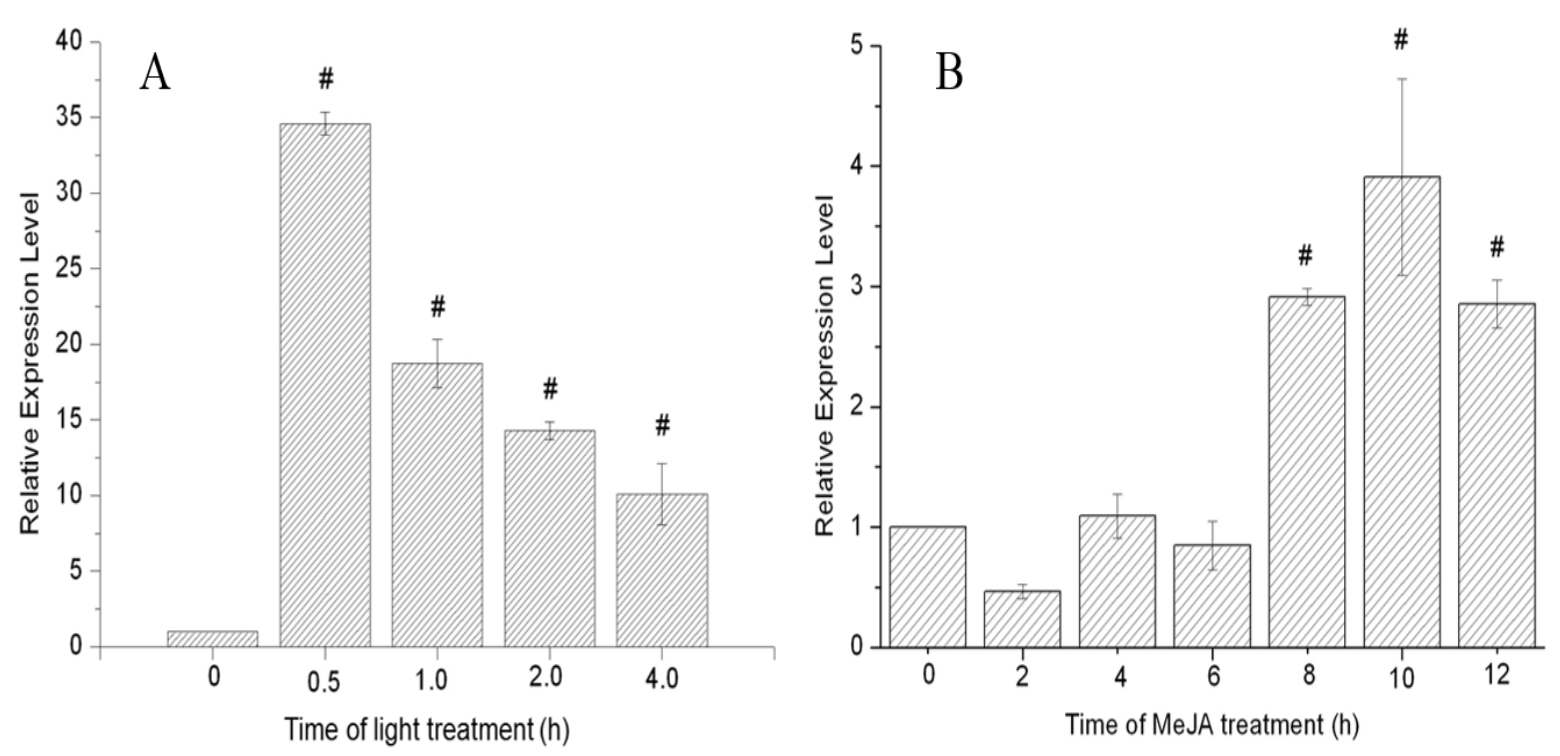

Figure 5. The expression of Cppsy gene induced by light (A) and MeJA (B) at different time points in $C$. protothecoides CS-41. Data (mean \pm SEM) are combined from three independent experiments. \# indicates that the gene expression levels were significantly different from that at $0 \mathrm{~h}(p<0.01)$.

\section{Experimental Section}

\subsection{Strains and Culture Conditions}

The microalgal strain used in this study was $C$. protothecoides CS-41 obtained from CSIRO Marine Laboratories (Hobart, Australia). They were grown in modified basal medium [32] containing $10 \mathrm{~g} \cdot \mathrm{L}^{-1}$ glucose at $28{ }^{\circ} \mathrm{C}$ and $180 \mathrm{rpm}$, and were collected at the log phase or late log phase.

\subsection{Genomic DNA and RNA Isolation}

Genomic DNA was extracted using a modified cetyltrimethylammonium bromide (CTAB) method [33]. The total RNA was isolated from $C$. protothecoides CS-41 cells at the late log phase (about

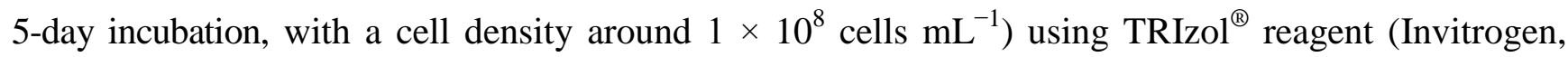
Carlsbad, CA, USA) according to the manufacturer's instructions.

\subsection{Cloning of Full-Length Cppsy cDNA and Its Corresponding Gene}

Degenerate primers (Table 1) were designed for the amplification of a partial Cppsy cDNA from C. protothecoides CS-41. The primers were derived from the highly conserved nucleotide and amino 
acid sequences reported for the psy genes from five kinds of algae (C. reinhardtii, H. pluvialis, D. salina, D. bardawil, and Chlorella NC_64A).

Sets of specific primers were synthesized based on the sequence of putative insert for 5'- and 3'-RACE [34]. YFO1 and YRI1 were used for 5'-RACE, and YFO2 and YRI2 (Table 1) were used for 3'-RACE. RACE was performed using the 5'-Full RACE Kit and 3'- Full RACE Core Set Version 2.0 (TaKaRa, Dalian, China) according to the manufacturer's protocol. The RACE products were gel purified and sequenced as previously described. One pair of specific primers, YF1 and YR1 (Table 1), was designed from the sequences of the 5'- and 3'-RACE fragments to amplify the full-length Cppsy cDNA and its corresponding gene.

\subsection{Southern Blot Analysis}

According to the Cppsy genomic DNA sequence, BamHI, EcoRI, NcoI, SmaI, and XmaI, which showed no recognition sites in the probed region of the Cppsy gene, were chosen to digest the whole genomic DNA. The probe was prepared by amplifying genomic DNA with the primers psy-F and psy-R, resulting in a 552-bp fragment of the Cppsy gene. The digested DNA was transferred to a Hybond-N membrane (GE Healthcare, Little Chanfont, UK) by capillary transfer and hybridized with a ${ }^{32} \mathrm{P}$-labelled DNA probe at both low and high stringency overnight.

After hybridization, the radioactivity of the membrane was monitored using a Storm 840 Phosphor Imaging System (Molecular Dynamics, Sunnyvale, CA, USA).

\subsection{Bioinformatics Analysis}

Comparative and bioinformatic analyses of the nucleotide sequences and deduced amino acid sequences were carried out online at http://www.ncbi.nlm.nih.gov and http://cn.expasy.org. The nucleotide sequence, deduced amino acid sequence, and open reading frame (ORF) were analyzed, and sequence comparison was conducted through database searches using BLAST programs (http://www.ncbi.nlm.nih.gov/BLAST/) and GeneDoc software. The phylogenetic analysis of psy from other plant species was aligned with Clustal X program version 1.83 using default parameters [35] and manual adjustments where necessary. A phylogenetic tree was constructed using MEGA (molecular evolutionary genetics analysis) program, version 4.0 [36] from Clustal W1.6 alignments. The NJ [37] method was used to construct the tree. In the NJ method, the P distance was used to analyze the amino acid sequences. A total of 1000 repetitions were performed using the bootstrap method to determine the reliability of each node of the tree. The homology-based three-dimensional structural modeling of PSY was accomplished using Swiss-Model and WebLab Viewer Lite (http://swissmodel.expasy.org).

\subsection{Functional Complementation Experiment in E. coli}

E. coli JM109 (Table 2) was used as a host for complementation experiments by cotransformation of the plasmid pUC-psy with pACCRT-E (Table 2). E. coli JM109 harboring only plasmid pACCRT-EB (Table 2) was cultured as a positive control, and only plasmid pUC-psy was cultured as a negative control for PSY functional analysis. The different strains were cultivated in $100 \mathrm{~mL}$ LB medium containing $100 \mu \mathrm{g} \cdot \mathrm{mL}^{-1}$ ampicillin and $50 \mu \mathrm{g} \cdot \mathrm{mL}^{-1}$ chloramphenicol at $28{ }^{\circ} \mathrm{C}$ and $180 \mathrm{rpm}$. IPTG 
$(1 \mathrm{mM})$ was added when the optical density at $600 \mathrm{~nm}\left(\mathrm{OD}_{600}\right)$ reached 0.5 , and the culture was kept at $28{ }^{\circ} \mathrm{C}$ for 2 days. The E. coli cells were collected by centrifugation at $12,000 \mathrm{rpm}$ and used for high-performance liquid chromatography (HPLC) analysis.

Table 2. Strains and plasmids used in this study.

\begin{tabular}{|c|c|c|}
\hline $\begin{array}{l}\text { Strains or } \\
\text { Plasmids }\end{array}$ & Characteristics & Source \\
\hline E. coli JM109 & Host for expression vectors & $\begin{array}{c}\text { MOST-USDA Joint Research Center for } \\
\text { Food Safety stock }\end{array}$ \\
\hline pACCRT-E & $\begin{array}{l}\text { pACYC184 containing crtE gene of } \\
\text { Erwinia uredovora }\left(\mathrm{Cm}^{\mathrm{r}}\right) \text { (metabolite: GGPP) }\end{array}$ & $\begin{array}{l}\text { Gift from Prof. Gerhard. Sandmann } \\
\text { (J.W. Goethe University, Frankfurt, } \\
\text { Germany) }\end{array}$ \\
\hline pACCRT-EB & $\begin{array}{l}\text { pACYC184 containing } c r t E \text { and } c t r B \text { genes of } \\
\text { Erwinia uredovora }\left(\mathrm{Cm}^{\mathrm{r}}\right) \text { (metabolite: Phytoene) }\end{array}$ & $\begin{array}{c}\text { Gift from Prof. Gerhard. Sandmann } \\
\text { (J.W. Goethe University, Frankfurt, } \\
\text { Germany) }\end{array}$ \\
\hline pUC19 & Expression vector $\left(A m p^{r}\right)$ & $\begin{array}{c}\text { MOST-USDA Joint Research Center for } \\
\text { Food Safety stock }\end{array}$ \\
\hline pUC-psy & pUC19 vector containing Cppsy gene $\left(\mathrm{Amp}^{\mathrm{r}}\right)$ & This work \\
\hline
\end{tabular}

Pigments in the bacteria were extracted according to procedures described by Breitenbach et al. [38]. E. coli JM109 cells harboring different plasmids were collected by centrifugation and freeze dried. Pigment extraction was carried out in acetone $(80 \%, \mathrm{v} / \mathrm{v})$ using ultrasonication, and the solvent was removed by blowing with nitrogen gas. The carotenoids were then resuspended in acetone for subsequent HPLC analysis. All operations were carried out on ice under dim light to prevent photodegradation, isomerizations, and structural changes of the carotenoids. The samples were prepared for HPLC by dissolving the dried residues in $1 \mathrm{~mL}$ of acetone and filtered through a polycarbonate $0.22 \mu \mathrm{m}$ filter (Millipore, Carrigtwohill, Ireland). The extracted pigments were separated on a Kromasil KR100-5C 18 analytical column $(250 \mathrm{~mm} \times 4.6 \mathrm{~mm}, 5 \mu \mathrm{m})$ using an UltiMate3000 HPLC system (Thermo Fisher Scientific, Waltham, MA, USA). The procedures described by Huang [39] were used with the following modifications: the mobile phase consisted of solvent A (acetonitrile/methanol/0.1 M Tris- $\mathrm{HCl}$ ( $\mathrm{pH}$ 8.0), 84:2:14, v/v/v) and solvent B (methanol/ethyl acetate, 68:32, v/v). Pigments were eluted at a flow rate of $1 \mathrm{~mL} \cdot \mathrm{min}^{-1}$ with a linear gradient from $100 \%$ solvent A to $100 \%$ solvent B over a $5 \mathrm{~min}$ period, followed by $25 \mathrm{~min}$ of solvent B. The column temperature was maintained at $30{ }^{\circ} \mathrm{C}$ and the sample volume was $20 \mu \mathrm{L}$. The pigments were monitored by diode array detector, and the targeted products were identified by their absorption spectra and typical retention times compared with the control.

\subsection{Promoter Isolation and Analysis}

The Genomic Walking Kit (TaKaRa, Dalian, China) was used to obtain promoter regions of the Cppsy gene from C. protothecoides CS-41. Based on the Cppsy genomic sequences, gene-specific primers were designed and are listed in Table 1. Primary and nested PCRs were performed with the Cppsy gene-specific primers and Genome Walking adapter primers (AP1) in the kit according to the manufacturer's instruction. The primary nested PCR products were diluted to 1:50 with distilled water 
for subsequent nested PCR. The nested PCR products were purified from 1.2\% (w/v) agarose gel and sub-cloned into the pMD18T vector (TaKaRa, Dalian, China). The cloned vectors were then sequenced and the putative cis-regulatory elements were analyzed using the PLACE [40] and PlantCARE databases [41].

\subsection{Gene Expression Response to Light and MeJA}

To analyze the light regulation pattern of the Cppsy gene in C. protothecoides, algal cells in the late $\log$ phase were cultivated in the dark for more than 2 days, then collected by centrifugation at $5000 \mathrm{rpm}$ for $15 \mathrm{~min}$ in the darkness. The pellet was resuspended in fresh medium without glucose, and then subjected to light treatment under a light intensity of $120 \mu \mathrm{mol} \mathrm{m} \mathrm{m}^{-2} \cdot \mathrm{s}^{-1}$ for different induction times $(0,0.5,1.0,2.0$, and $4.0 \mathrm{~h})$. Each treatment was carried out with three parallel repetitions.

To analyze the MeJA regulation pattern of the Cppsy gene in C. protothecoides, algal cells in the $\log$ phase were treated with $100 \mu \mathrm{M}$ MeJA (Sigma, St. Louis, MO, USA) diluted in dimethyl sulfoxide (DMSO) for 0, 2, 4, 6, 8 10, and $12 \mathrm{~h}$. Control cultures were treated with DMSO only.

The effects of light and MeJA on the Cppsy gene transcripts in C. protothecoides were quantified by reverse transcriptase quantitative PCR (RT-qPCR). The RT-qPCR experiment was performed in two steps: the cDNA templates were synthesized from RNA samples using Prime Script ${ }^{\mathrm{TM}}$ Reverse Transcriptase Reagent according to the manufacturer's instructions (TaKaRa, Dalian, China) using oligo (dT) as the primer; then qPCR was conducted on an iQ Cycler (Bio-Rad, Watford, UK) using the specific primers (Table 1) and the SYBR ExScript RT-PCR kit (TaKaRa, Dalian, China). The specific primers for the corresponding genes included psyRT-F and psyRT-R for the Cppsy gene, and 16SRT-F and 16SRT-R for the $16 \mathrm{~S}$ gene (Table 1). Before the qPCR expression analysis, we checked the amplification efficiency of each primer pairs, and all were well controlled between $99.83 \%$ and $101.25 \%$.

Each qPCR measurement was carried out independently at least three times, and the mean value was used for quantification. The $2^{-\Delta \Delta \mathrm{CT}}$ method was used to analyze the relative changes in gene expression, the expression of the $16 \mathrm{~S}$ gene was used as a normalized control, and the expression of the untreated samples was used as a negative control.

\section{Conclusions}

Carotenoid pathways in plants have been described in great detail using genetic, biochemical, and molecular data, mainly from Arabidopsis and other higher plants; however, this is the first study in the unicellular microalga $C$. protothecoides $\mathrm{CS}-41$.

We successfully isolated and analyzed the Cppsy gene, which encodes the functional phytoene synthase - a vital enzyme for carotenoid biosynthesis in C. protothecoides CS-41-as well as its promoter. Computational analysis suggested that this protein belongs to the Isoprenoid_Biosyn_C1 superfamily. It contains one more substrate- $\mathrm{Mg}^{2+}$ binding site than other algae and higher plants. Analysis also demonstrated several candidate motifs for the promoter, which exhibited light- and MeJA-responsive characteristics. Light- and MeJA treatment showed that the Cppsy expression level was significantly enhanced by light and MeJA. 
These achievements will be helpful to understand more about the regulatory mechanism of the carotenoid biosynthesis pathway in algae and the mechanisms for accumulation of lutein and other important carotenoids.

\section{Acknowledgments}

This work was supported by the National 863 Program of China (2012AA101601), and the Agri-X Program of Shanghai Jiao Tong University (Agri-X2015005).

\section{Author Contributions}

Chunlei Shi and Xianming Shi conceived and designed the experiments. Meiya Li, Yan Cui and Zhibing Gan performed the experiments. Chunlei Shi, Meiya Li and Yan Cui analyzed the data and prepared the manuscript.

\section{Conflict of Interest}

The authors declare no conflict of interest.

\section{References}

1. Demmig-Adams, B.; Adams, W.W. Antioxidants in photosynthesis and human nutrition. Science 2002, 298, 2149-2153.

2. Vishwanathan, R.; Kuchan, M.J.; Sen, S.; Johnson, E.J. Lutein and Preterm Infants with Decreased Concentrations of Brain Carotenoids. J. Pediatr. Gastroenterol. Nutr. 2014, 59, 659-665.

3. Perrone, S.; Tei, M.; Longini, M.; Santacroce, A.; Turrisi, G.; Proietti, F.; Felici, C.; Picardi, A.; Bazzini, F.; Vasarri, P.; et al. Lipid and protein oxidation in newborn infants after lutein administration. Oxid. Med. Cell. Longev. 2014, 2014, doi:10.1155/2014/781454.

4. Kalariya, N.M.; Ramana, K.V.; Vankuijk, F.J. Focus on molecules: Lutein. Exp. Eye Res. 2012, 102, 107-108.

5. Widomska, J.; Subczynski, W.K. Why has Nature Chosen Lutein and Zeaxanthin to Protect the Retina? J. Clin. Exp. Ophthalmol. 2014, 5, 326.

6. Mares-Perlman, J.A.; Millen, A.E.; Ficek, T.L.; Hankinson, S.E. The body of evidence to support a protective role for lutein and zeaxanthin in delaying chronic disease: Overview. J. Nutr. 2002, $132,518 \mathrm{~S}-524 \mathrm{~S}$.

7. Kim, H.W.; Chew, B.P.; Wong, T.S.; Park, J.S.; Weng, B.B.; Byrne, K.M.; Hayek, M.G.; Reinhart, G.A. Dietary lutein stimulates immune response in the canine. Vet. Immunol. Immunopathol. 2000, 74, 315-327.

8. Krinsky, N.I.; Landrum, J.T.; Bone, R.A. Biologic mechanisms of the protective role of lutein and zeaxanthin in the eye. Annu. Rev. Nutr. 2003, 23, 171-201.

9. Granado, F.; Olmedilla, B.; Blanco, I. Nutritional and clinical relevance of lutein in human health. Annu. Rev. Nutr. 2003, 90, 487-502.

10. Maia, M.; Furlani, B.A.; Souza-Lima, A.A.; Martins, D.S.; Navarro, R.M.; Belfort, R.J. Lutein: A new dye for chromovitrectomy. Retina 2014, 34, 262-272. 
11. Shi, X.M.; Zhang, X.W.; Chen, F. Heterotrophic production of biomass and lutein by Chlorella protothecoides on various nitrogen sources. Enzyme Microb. Technol. 2000, 27, 312-318.

12. Shi, X.M.; Jiang, Y.; Chen, F. High-yield production of lutein by the green microalga Chlorella protothecoides in heterotrophic fed-batch culture. Biotechnol. Prog. 2002, 18, 723-727.

13. Shi, X.M.; Wu, Z.Y.; Chen, F. Kinetic modeling of lutein production by heterotrophic Chlorella at various $\mathrm{pH}$ and temperatures. Mol. Nutr. Food Res. 2006, 50, 763-768.

14. Chen, Q.; Jiang, J.G.; Wang, F. Molecular phylogenies and evolution of crt genes in algae. Crit. Rev. Biotechnol. 2007, 27, 77-91.

15. Salvini, M.; Bernini, A.; Fambrini, M.; Pugliesi, C. cDNA cloning and expression of the phytoene synthase gene in sunflower. J. Plant Physiol. 2005, 162, 479-484.

16. Li, F.; Vallabhaneni, R.; Yu, J.; Rocheford, T.; Wurtzel, E.T. The maize phytoene synthase gene family: Overlapping roles for carotenogenesis in endosperm, photomorphogenesis, and thermal stress tolerance. Plant Physiol. 2008, 147, 1334-1346.

17. Bohne, F.; Linden, H. Regulation of carotenoid biosynthesis genes in response to light in Chlamydomonas reinhardtii. Biochim. Biophys. Acta 2002, 1579, 26-34.

18. Lao, Y.M.; Xiao, L.; Ye, Z.W.; Jiang, J.G.; Zhou, S.S. In silico analysis of phytoene synthase and its promoter reveals hints for regulation mechanisms of carotenogenesis in Duanliella bardawil. Bioinformatics 2011, 27, 2201-2208.

19. Steinbrenner, J.; Linden, H. Regulation of two carotenoid biosynthesis genes coding for phytoene synthase and carotenoid hydroxylase during stress-induced astaxanthin formation in the green alga Haematococcus pluvialis. Plant Physiol. 2001, 125, 810-817.

20. McCarthy, S.S.; Kobayashi, M.C.; Niyogi, K.K. White mutants of Chlamydomonas reinhardtii are defective in phytoene synthase. Genetics 2004, 168, 1249-1257.

21. Steinbrenner, J.; Linden, H. Light induction of carotenoid biosynthesis genes in the green alga Haematococcus pluvialis: Regulation by photosynthetic redox control. Plant Mol. Biol. 2003, 52, 343-356.

22. Couso, I.; Vila, M.; Rodriguez, H.; Vargas, M.A.; Leon, R. Overexpression of an exogenous phytoene synthase gene in the unicellular alga Chlamydomonas reinhardtii leads to an increase in the content of carotenoids. Biotechnol. Prog. 2011, 27, 54-60.

23. Cordero, B.F.; Couso, I.; Leon, R.; Rodriguez, H.; Vargas, M.A. Enhancement of carotenoids biosynthesis in Chlamydomonas reinhardtii by nuclear transformation using a phytoene synthase gene isolated from Chlorella zofingiensis. Appl. Microbiol. Biotechnol. 2011, 91, 341-351.

24. Li, M.Y.; Gan, Z.B.; Cui, Y.; Shi, C.L.; Shi, X.M. Structure and function characterization of the phytoene desaturase related to the lutein biosynthesis in Chlorella protothecoides CS-41. Mol. Biol. Rep. 2013, 40, 3351-3361.

25. Li, M.Y.; Gan, Z.B.; Cui, Y.; Shi, C.L.; Shi, X.M. Cloning and characterization of the zeta-carotene desaturase gene from Chlorella protothecoides CS-41. J. Biomed. Biotechnol. 2011, 2011, doi:10.1155/2011/731542.

26. Gallagher, C.E.; Matthews, P.D.; Li, F.; Wurtzel, E.T. Gene duplication in the carotenoid biosynthetic pathway preceded evolution of the grasses. Plant Physiol. 2004, 135, 1776-1783. 
27. Li, F.; Vallabhaneni, R.; Wurtzel, E. PSY3, a new member of the phytoene synthase gene family conserved in the Poaceae and regulator of abiotic stress-induced root carotenogenesis. Plant Physiol. 2008, 146, 1333-1345.

28. Welsch, R.; Wust, F.; Bar, C.; al-Babili, S.; Beyer, P. A third phytoene synthase is devoted to abiotic stress-induced abscisic acid formation in rice and defines functional diversification of phytoene synthase genes. Plant Physiol. 2008, 147, 367-380.

29. Tran, D.; Haven, J.; Qiu, W.G.; Polle, J.E. An update on carotenoid biosynthesis in algae: Phylogenetic evidence for the existence of two classes of phytoene synthase. Planta 2009, 229, 723-729.

30. Joshi, C.P. An inspection of the domain between putative TATA box and translation start site in 79 plant genes. Nucleic Acids Res. 1987, 15, 6643-6653.

31. Cazzonelli, C.I.; Pogson, B.J. Source to sink: Regulation of carotenoid biosynthesis in plants. Trends Plant Sci. 2010, 15, 266-274.

32. Shi, X.M.; Chen, F.; Yuan, J.-P.; Chen, H. Heterotrophic production of lutein by selected Chlorella strains. J. Appl. Phycol. 1997, 9, 445-450.

33. Stewart, C.J.; Via, L.E. A rapid CTAB DNA isolation technique useful for RAPD fingerprinting and other PCR applications. BioTechniques 1993, 14, 748-750.

34. Yeku, O.; Frohman, M.A. Rapid amplification of cDNA ends (RACE). In RNA; Nielsen, H., Ed.; Humana Press: Totowa, NJ, USA, 2011; pp. 107-122.

35. Thompson, J.D.; Gibson, T.J.; Plewniak, F.; Jeanmougin, F.; Higgins, D.G. The CLUSTAL_X windows interface: Flexible strategies for multiple sequence alignment aided by quality analysis tools. Nucleic Acids Res. 1997, 25, 4876-4882.

36. Tamura, K.; Dudley, J.; Nei, M.; Kumar, S. MEGA4: Molecular Evolutionary Genetics Analysis (MEGA) software version 4.0. Mol. Biol. Evol. 2007, 24, 1596-1599.

37. Saitou, N.; Nei, M. The neighbor-joining method: A new method for reconstructing phylogenetic trees. Mol. Biol. Evol. 1987, 4, 406-425.

38. Breitenbach, J.; Zhu, C.; Sandmann, G. Bleaching herbicide norflurazon inhibits phytoene desaturase by competition with the cofactors. J. Agric. Food Chem. 2001, 49, 5270-5272.

39. Huang, J.C.; Liu, J.; Li, Y.T.; Chen, F. Isolation and characterization of the phytoene desaturase gene as a potential selective marker for genetic engineering of the astaxanthin-producing green alga Chlorella zofingiensis (Chlorophyta). J. Phycol. 2008, 44, 684-690.

40. Higo, K.; Ugawa, Y.; Iwamoto, M.; Korenaga, T. Plant cis-acting regulatory DNA elements (PLACE) database: 1999. Nucleic Acids Res. 1999, 27, 297-300.

41. Lescot, M.; Dehais, P.; Thijs, G.; Marchal, K.; Moreau, Y.; van de Peer, Y.; Rouze, P.; Rombauts, S. PlantCARE, a database of plant cis-acting regulatory elements and a portal to tools for in silico analysis of promoter sequences. Nucleic Acids Res. 2002, 30, 325-327.

(C) 2015 by the authors; licensee MDPI, Basel, Switzerland. This article is an open access article distributed under the terms and conditions of the Creative Commons Attribution license (http://creativecommons.org/licenses/by/4.0/). 